\title{
Cálculo de Autovalores via Métodos tipo Newton
}

L.H. BEZERRA, Departamento de Matemática, UFSC, 88040-900 Florianópolis, $\mathrm{SC}$, Brasil.

Resumo. Neste trabalho, desenvolve-se uma análise numérica de alguns métodos de decomposição parcial do espectro de uma matriz, mostrando a relação desses métodos com o método de Newton para calcular zeros de funções. Um desses métodos é o DPSE (Dominant Pole Spectrum Eigensolver), que surgiu em problemas de Controle. Provamos nesse trabalho que esse método, que é tipo Newton, tem convergência local quadrática. São mostrados alguns resultados interessantes obtidos da aplicação do DPSE ao problema de estabilidade local do sistema elétrico de potência brasileiro, que são então comparados a resultados obtidos com métodos tradicionais utilizados também na resolução desse problema.

\section{Introdução}

O problema de autovalores é um dos problemas centrais da Álgebra Linear. Jacobi foi pioneiro na formulação de um método iterativo para calcular o espectro de uma matriz simétrica, isso em 1846. Hoje em dia, o sistema iterativo MATLAB tem a função eig para calcular o espectro de uma matriz genérica via método $Q R$, método surgido no início da segunda metade do século XX. A convergência deste método para matrizes não normais pode ser problemática, por exemplo, se alguns dos autovalores estiverem agrupados, muito próximos um do outro. Nos problemas práticos, porém, interessa-nos apenas calcular alguns autovalores, não todos. Por exemplo, em análise de estabilidade local de sistemas dinâmicos, estamos interessados em calcular autovalores com parte real positiva ou aqueles que, tendo parte real negativa, estão muito próximos do eixo imaginário (esses autovalores poderiam ser autovalores de parte real positiva, se não houvessem erros nos dados traduzidos para a matriz do sistema). Uma versão prática dessa análise é a análise de estabilidade de um sistema elétrico de potência a pequenas perturbações, que procura identificar as causas de oscilações instáveis ou fracamente amortecidas do sistema (autovalores com parte real positiva ou quase). Para isso, as equações não lineares que modelam o comportamento dinâmico do sistema são linearizadas para uma vizinhança de um ponto de operação. Uma análise modal, baseada na computação dos autovalores relevantes, e respectivos autovetores (que leva em conta, ainda, a sensibilidade do cálculo destes à perturbação dos dados), é então utilizada para a análise e controle da estabilidade do sistema. Enfim, o cálculo do espectro parcial de uma matriz é essencial, e não só do ponto de vista matemático.

Neste trabalho, vamos mostrar a conexão que alguns métodos de cálculo parcial de espectro têm com o método de Newton para calcular zeros de funções. Vamos 
mostrar também que um método muito utilizado na área de Controle para calcular polos dominantes de funções de transferência, o DPSE (Dominant Pole Spectrum Eigensolver [7], [9]), é um método de cálculo de autovalores que converge localmente de forma quadrática. Finalmente, apresentamos alguns resultados obtidos, via DPSE, com dois modelos práticos: um, modelo do sistema sul-sudeste brasileiro de potência (citenm); outro, modelo do sistema norte-sul brasileiro, que foi interconectado em 1999 ([4]). Comparamos então esses resultados com os obtidos via método de potências inversas e via método de Arnoldi, que são utilizados correntemente na resolução do problema de estabilidade local de sistemas de potência [7].

A organização desse artigo é a seguinte: na seção 2, mostramos que os métodos de potência inversa e o de polo dominante geram sequências análogas (ou idênticas) às que o método de Newton gera para funções particulares. Na seção 3, apresentamos a prova de que o DPSE é um método de cálculo de espectro parcial que tem convergência local quadrática. Finalmente, na seção 4, comparamos resultados numéricos obtidos por DPSE com resultados obtidos pelo método de potências inversas com deslocamento (INVIT) e com os obtidos pelo método de Arnoldi (função eigs do MATLAB).

\section{Calculando um autovalor}

Vamos considerar que uma matriz $A$ tenha autovalores distintos e que $\lambda$ seja o autovalor mais próximo de um valor complexo $\mu$. Suponhamos que o autovetor associado a $\lambda$ tenha a coordenada de maior valor absoluto igual a 1 e que essa coordenada seja a enésima. A seguir apresentamos uma proposição, cuja demonstração usa técnicas básicas de Álgebra Linear, o que a torna mais simples e direta que a prova apresentada em [10].

Proposição 2.1. O método de Newton para a função

$$
f(x)=A x-x e_{n}^{T} A x, \quad e_{n}^{T} x=1,
$$

gera, a partir de um vetor $x_{0}$ tal que $e_{n}^{T} x_{0}=1$ e de $\lambda_{0}=e_{n}^{T} A x_{0}=\mu$, os mesmos vetores que o método da potência inversa geraria (a menos de normalização), a partir desses dados iniciais.

Prova. A derivada de $f$ em $x_{0}$ é dada por

$$
f^{\prime}\left(x_{0}\right) . z=A z-z e_{n}^{T} A x_{0}-x_{0} e_{n}^{T} A z=\left(A-\lambda_{0} I-x_{0} e_{n}^{T} A\right) z .
$$

Pelo método de Newton,

$$
\begin{aligned}
x_{1} & =x_{0}-\left(A-\lambda_{0} I-x_{0} e_{n}^{T} A\right)^{-1} f\left(x_{0}\right) \\
& =\left(A-\lambda_{0} I-x_{0} e_{n}^{T} A\right)^{-1}\left[\left(A-\lambda_{0} I-x_{0} e_{n}^{T} A\right) x_{0}-A x_{0}+x_{0} e_{n}^{T} A x_{0}\right] \\
& =-\lambda_{0}\left(A-\lambda_{0} I-x_{0} e_{n}^{T} A\right)^{-1} x_{0}
\end{aligned}
$$

Pela fórmula de Shermann-Morrison,

$$
\begin{aligned}
x_{1} & =-\left[\left(A-\lambda_{0} I\right)^{-1}+\frac{\left(A-\lambda_{0} I\right)^{-1} x_{0} e_{n}^{T} A\left(A-\lambda_{0} I\right)^{-1}}{1-e_{n}^{T} A x_{0}}\right] x_{0} \\
& =-\left(\lambda_{0}+\frac{\lambda_{0} z_{1}}{1-\lambda_{0}}\right)\left(A-\lambda_{0}\right)^{-1} x_{0},
\end{aligned}
$$


em que $z_{1}=e_{n}^{T} A\left(A-\lambda_{0} I\right)^{-1} x_{0}$.

Assim, $x_{1}$ pertence ao subespaço gerado por $\left(A-\lambda_{0} I\right)^{-1} x_{0}$, que é o vetor dado pela iteração inversa, antes de ser normalizado.

Um método muito utilizado para calcular um polo dominante de uma função de transferência do tipo

$$
H(s)=c^{T}(A-s I)^{-1} b
$$

é o DPA (Dominant Pole Algorithm, [8]): dada uma aproximação inicial $s_{0}$,

$$
s_{k}=\frac{y_{k}^{T} A x_{k}}{y_{k}^{T} x_{k}},
$$

em que $x_{k}=\left(A-s_{k-1} I\right)^{-1} b$ e $y_{k}=\left(A^{T}-s_{k-1} I\right)^{-1} c$. Como $A x_{k}=\left(A-s_{k-1} I+\right.$ $\left.s_{k-1} I\right)\left(A-s_{k-1} I\right)^{-1} b=b+s_{k-1}\left(A-s_{k-1} I\right)^{-1} b$,

$$
s_{k}=s_{k-1}+\frac{y_{k}^{T} b}{y_{k}^{T} x_{k}}=s_{k-1}+\frac{c^{T}\left(A-s_{k-1} I\right)^{-1} b}{c^{T}\left(A-s_{k-1} I\right)^{-2} b} .
$$

Mas, esta é a expressão da iteração de Newton para calcular zeros da função

$$
L(s)=\frac{1}{H(s)} \text {. }
$$

\section{Calculando alguns autovalores}

Nesta seção, vamos introduzir o DPSE (Dominant Pole Spectrum Eigensolver), método que calcula simultaneamente vários polos da função de transferência $H(s)=$ $c^{T}(A-s I)^{-1} b[7]$.

Vamos supor daqui por diante que $A \in \mathbb{R}^{N \times N}$ é uma matriz com $N$ autovalores distintos, e que $b, c \in \mathbb{R}^{N}$, são tais que $\|b\|_{2}=\|c\|_{2}=1$.

Consideremos, agora, que $s_{1}^{(k)}, \ldots, s_{p}^{(k)}$ são $p$ escalares calculados no passo $k$. Definamos, para cada $1 \leq i \leq p$, os vetores $x_{i}^{(k)}$ e $y_{i}^{(k)}$ por

$$
x_{i}^{(k)}=\frac{\left(A-s_{i}^{(k)} I\right)^{-1} b}{c^{T}\left(A-s_{i}^{(k)} I\right)^{-1} b} \quad \text { e } \quad y_{i}^{(k)}=\frac{\left(A^{T}-s_{i}^{(k)} I\right)^{-1} c}{c^{T}\left(A-s_{i}^{(k)} I\right)^{-1} b} i .
$$

Sejam $X=X^{(k)}$ e $Y=Y^{(k)}$ as matrizes $N \times p$, cujas colunas são, respectivamente, os vetores $x_{1}^{(k)}, \ldots, x_{p}^{(k)}$ e $y_{1}^{(k)}, \ldots, y_{p}^{(k)}$. Seja $M=M^{(k)}=\left(Y^{T} X\right)^{-1}\left(Y^{T} A X\right)$. Pelo DPSE, o passo seguinte é dado por

$$
\left\{s_{1}^{(k+1)}, \ldots, s_{p}^{(k+1)}\right\}=\lambda(M) .
$$

A seguir, apresentamos alguns resultados teóricos preliminares.

Considere-se $A=P D P^{-1}$, a decomposição espectral de $A$ tal que $c^{T}(A-$ $s I)^{-1} b=\sum_{i=1}^{N} \frac{r_{i}}{\lambda_{i}-s}$, com $\left|r_{1}\right| \geq \cdots \geq\left|r_{p}\right|>>\left|r_{p+1}\right| \geq \cdots\left|r_{N}\right|$, em que $r_{i}=$ $e_{i}^{T} P^{-1} b c^{T} P e_{i}$, e $\lambda_{i}=D_{i i}$. Ou seja, denotando os vetores colunas de $P$ por 
$u_{1}, \cdots, u_{N}$, e os vetores linhas de $P^{-1}$ por $v_{1}, \cdots, v_{N}$, temos que $b=\sum_{i=1}^{N} b_{i} u_{i}$ e $c=\sum_{i=1}^{N} c_{i} v_{i}$, para certos escalares $b_{i}, c_{i}, 1 \leq i \leq N$. Ou seja, $r_{i}=b_{i} c_{i}$.

Seja $S=\left(s_{1} \cdots s_{p}\right)^{T} \in \mathbb{C}^{p}$, tal que $s_{i} \neq s_{j}$, se $i \neq j$, e, para todo $i, s_{i}$ não seja um autovalor de $A$. Para $i=1, \ldots, p$ sejam

$$
x_{i}=\frac{\left(A-s_{i} I\right)^{-1} b}{c^{T}\left(A-s_{i} I\right)^{-1} b}, \quad y_{i}=\frac{\left(A^{T}-s_{i} I\right)^{-1} c}{c^{T}\left(A-s_{i} I\right)^{-1} b}
$$

Observemos que

$$
x_{i}=\frac{A d j\left(A-s_{i} I\right) b}{c^{T} A d j\left(A-s_{i} I\right) b}, \quad y_{i}=\frac{\operatorname{Adj}\left(A^{T}-s_{i} I\right) c}{c^{T} \operatorname{Adj}\left(A-s_{i} I\right) b}
$$

e, assim, $x_{i}$ e $y_{i}$ estão bem definidos quando $s_{i}$ é um autovalor simples de $A$, pois, neste caso, $A d j\left(A-s_{i} I\right)=\hat{u}_{i} \hat{v}_{i}^{H}$, em que $\hat{u}_{i}$ e $\hat{v}_{i}$ são, respectivamente, autovetores à direita e à esquerda de $A$ associados a $s_{i}$. Sejam $X=\left[x_{1} \cdots x_{p}\right]$ e $Y=\left[y_{1} \cdots y_{p}\right]$ (como definidos anteriormente).

Lema 3.1. A matriz $Y^{T} X$ é simétrica e inversível.

Prova.

$$
\begin{aligned}
\left(Y^{T} X\right)_{i j} & =e_{i}^{T} Y^{T} X e_{j}=\frac{c^{T}\left(A-s_{i} I\right)^{-1}}{c^{T}\left(A-s_{i} I\right)^{-1} b} \frac{\left(A-s_{j} I\right)^{-1} b}{c^{T}\left(A-s_{j} I\right)^{-1} b} \\
& =\frac{c^{T}\left(A-s_{j} I\right)^{-1}}{c^{T}\left(A-s_{j} I\right)^{-1} b} \frac{\left(A-s_{i} I\right)^{-1} b}{c^{T}\left(A-s_{i} I\right)^{-1} b}
\end{aligned}
$$

Para mostrar que $Y^{T} X$ é não singular, observe-se que $Y^{T} X=F G^{T} R G F$, em que $G \in \mathbb{C}^{N \times p}$ é tal que $G_{i j}=\left(\lambda_{i}-s_{j}\right)^{-1}$ e $F$ e $R$ são matrizes diagonais, $N \times N$ : $F_{i i}=\frac{1}{c^{T}\left(A-s_{i} I\right)^{-1} b}$ e $R_{i i}$ é o resíduo $r_{i}$. Como $\left|r_{1}\right|, \ldots,\left|r_{p}\right|$ são não nulos, o posto de $R$ é, no mínimo, $p$. A matriz $F$ é não singular (para todo $i, s_{i}$ não é autovalor de $A$ ). Além disso, como a submatriz $G(1: p, 1: p)$ é não singular (matriz de Cauchy [1]), o posto de $Y^{T} X$ é $p$.

Observação. Na prática, $x_{i}, y_{i}$ são calculados do seguinte modo: primeiro, normalizam-se $\left(A-s_{i} I\right)^{-1} b,\left(A-s_{i} I\right)^{-T} c$, o que resulta nos vetores $z_{i}, w_{i}$, respectivamente; ambos são então divididos por $c^{T} z_{i}$, que é igual a $b^{T} w_{i}$. Este procedimento evita erros numéricos quando o método começa a convergir. É importante notar que, em aritmética exata, os resultados são idênticos, normalizando-se primeiro ou não os vetores.

Seja $M=\left(Y^{T} X\right)^{-1} Y^{T} A X$. Como

$$
A X e_{i}=A \frac{\left(A-s_{i} I\right)^{-1} b}{c^{T}\left(A-s_{i} I\right)^{-1} b}=s_{i} X e_{i}+\frac{b}{c^{T}\left(A-s_{i} I\right)^{-1} b},
$$

concluímos que

$$
M=\operatorname{diag}(S)+\left(Y^{T} X\right)^{-1} Y^{T} B
$$


em que $\operatorname{diag}(S)$ é a matriz diagonal construída a partir de $S$, e $B$ é a matriz tal que, para todo $i, B e_{i}=b / c^{T}\left(A-s_{i} I\right)^{-1} b$. Ou seja, $M$ é uma perturbação de posto 1 da matriz $\operatorname{diag}(S)$, o que significa que $M$ é uma matriz diagonalizável perto de $S$.

Consideremos, agora, uma decomposição espectral de $M: M=Q D Q^{-1}$. Vamos definir a seguinte função: $F(S)=\left(F_{1}(S), \ldots, F_{p}(S)\right)$, em que

$$
F_{i}(S)=s_{i}+\frac{c^{T} X Q e_{i}}{c^{T}\left(A-s_{i} I\right)^{-1} X Q e_{i}} .
$$

Como $c^{T}\left(A-s_{i} I\right)^{-1} A=c^{T}\left(A-s_{i} I\right)^{-1}\left(A-s_{i} I+s_{i} I\right)=c^{T}+s_{i} c^{T}\left(A-s_{i} I\right)^{-1}, \mathrm{e}$ $c^{T}\left(A-s_{i} I\right)^{-1} A X Q e_{i}=d_{i i} c^{T}\left(A-s_{i} I\right)^{-1} X Q e_{i}$, obtemos, então, que

$$
c^{T} X Q e_{i}=\left(d_{i i}-s_{i}\right) c^{T}\left(A-s_{i} I\right)^{-1} X Q e_{i} .
$$

Por conseguinte,

$$
F_{i}(S)=d_{i i}
$$

(Note-se que esta função está realmente bem definida no espaço quociente $\mathbb{C}^{n} / \sim$, em que $x \sim y$ se, e somente se, existe uma matriz de permutação $P$ tal que $y=P x$.)

Se $s_{i}$ é um autovalor de $A$, vamos definir $F_{i}(S)=s_{i}$. Se $S=\left(s_{1} \cdots s_{p}\right) \in \mathbb{C}^{p}$, com $s_{i} \neq s_{j}$ se $i \neq j$, então $M$ é uma matriz que depende analiticamente de $S$. Logo, $F$ também depende analiticamente de $S$ (ou, rigorosamente falando, da classe de equivalência de $S$ ). O teorema seguinte afirma que se $\lambda_{1}, \ldots, \lambda_{p}$ são autovalores de $A$, distintos dois a dois, então $\left(\lambda_{1}, \ldots, \lambda_{p}\right)$ é um ponto fixo de $F$.

Teorema 3.1. Sejam $\lambda_{1}, \ldots, \lambda_{p}$ autovalores de $A$, distintos dois a dois. Se $s_{1}^{(k)} \rightarrow$ $\lambda_{1}, \ldots, s_{p}^{(k)} \rightarrow \lambda_{p}$, então $F\left(s_{1}^{(k)}, \ldots, s_{p}^{(k)}\right) \rightarrow\left(\lambda_{1}, \ldots, \lambda_{p}\right)$.

Prova.

$$
\begin{aligned}
& x_{k}^{(k)}=\frac{\left(A-s_{i}^{(k)} I\right)^{-1} b}{c^{T}\left(A-s_{i}^{(k)} I\right)^{-1} b}=\frac{\operatorname{Adj}\left(A-s_{i}^{(k)} I\right) b}{c^{T} \operatorname{Adj}\left(A-s_{i}^{(k)} I\right) b} \\
& y_{k}^{(k)}=\frac{\left(A-s_{i}^{(k)} I\right)^{-T} c}{c^{T}\left(A-s_{i}^{(k)} I\right)^{-1} b}=\frac{\left(A d j\left(A-s_{i}^{(k)} I\right)\right)^{T} b}{c^{T} \operatorname{Adj}\left(A-s_{i}^{(k)} I\right) b} .
\end{aligned}
$$

Quando $k \rightarrow \infty, x_{i}^{(k)} \rightarrow \frac{u_{i}}{c^{T} u_{i}}$, e $y_{i}^{(k)} \rightarrow \frac{v_{i}}{b^{T} v_{i}}$. Logo, $\left(Y_{k}^{T} X_{k}\right)^{-1} Y_{k}^{T} A X_{k} \rightarrow$ $\left(\lambda_{1}, \ldots, \lambda_{p}\right)$.

Teorema 3.2. DPSE tem convergência local quadrática.

Prova. Podemos verificar que o DPSE é definido por $S^{(k+1)}=F\left(S^{(k)}\right)$, em que $F$ é a função definida acima. É suficiente, então, mostrar que $D F(\Lambda)=0$, em que $\Lambda=\left(\lambda_{1}, \ldots, \lambda_{p}\right)$. Agora

$$
\begin{aligned}
\frac{\partial F_{i}}{\partial s_{j}}(\Lambda)= & \delta_{i j}+\operatorname{det}\left(A-\lambda_{i} I\right) \frac{c^{T} \frac{\partial}{\partial s_{j}}\left(X Q e_{i}\right)}{c^{T} A d j\left(A-\lambda_{i} I\right) X Q e_{i}} \\
& -\delta_{i j} \frac{c^{T} X Q e_{i}}{\left(c^{T} A d j\left(A-\lambda_{i} I\right) X Q e_{i}\right)^{2}} c^{T}\left(A d j\left(A-\lambda_{i} I\right)\right)^{2} X Q e_{i} \\
& -\operatorname{det}\left(A-\lambda_{i} I\right) \frac{c^{T} X Q e_{i}}{\left(c^{T} A d j\left(A-\lambda_{i} I\right) X Q e_{i}\right)^{2}} c^{T} A d j\left(A-\lambda_{i} I\right) \frac{\partial}{\partial s_{j}}\left(X Q e_{i}\right)
\end{aligned}
$$


e, como $\operatorname{det}\left(A-\lambda_{i} I\right)=0$, para $\lambda_{i} \in \lambda(A)$

$$
\frac{\partial F_{i}}{\partial s_{j}}(\Lambda)=\delta_{i j}-\delta_{i j} \frac{c^{T} X Q e_{i}}{\left(c^{T} A d j\left(A-\lambda_{i} I\right) X Q e_{i}\right)^{2}} c^{T}\left(\operatorname{Adj}\left(A-\lambda_{i} I\right)\right)^{2} X Q e_{i},
$$

em que $\delta_{i j}=1$ se $i=j$, e zero, caso contrário. Mas, quando $\lambda_{i} \in \lambda(A)$,

$$
\begin{aligned}
\frac{c^{T} X Q e_{i}}{\left(c^{T} A d j\left(A-\lambda_{i} I\right) X Q e_{i}\right)^{2}} c^{T}\left(\operatorname{Adj}\left(A-\lambda_{i} I\right)\right)^{2} X Q e_{i} & =\frac{c^{T} u_{i}}{\left(c^{T} \hat{u}_{i} \hat{v}_{i}^{H} u_{i}\right)^{2}} c^{T}\left(\hat{u}_{i} \hat{v}_{i}^{H}\right)^{2} u_{i} \\
& =1 .
\end{aligned}
$$

Ou seja, $\frac{\partial F_{i}}{\partial s_{j}}(\Lambda)=0$

\section{Testes e comparações}

Um sistema elétrico de potência pode ser descrito por um sistema não linear de equações diferenciais e algébricas do tipo

$$
\left\{\begin{array}{l}
\dot{x}=f(x, y) \\
0=g(x, y)
\end{array}\right.
$$

em que $x$, o vetor de estado, contém as variáveis dinâmicas e y, as variáveis algébricas. Após linearização em torno de um ponto de operação do sistema, isto é, um ponto $\left(x_{0}, y_{0}\right)$ tal que $\mathrm{f}\left(x_{0}, y_{0}\right)=0$, obtemos o sistema seguinte:

$$
\left(\begin{array}{c}
\Delta \dot{x} \\
0
\end{array}\right)=\left(\begin{array}{cc}
J_{1} & J_{2} \\
J_{3} & J_{4}
\end{array}\right)\left(\begin{array}{c}
\Delta x \\
\Delta y
\end{array}\right)
$$

A matriz do sistema acima é dita a jacobiana do sistema. Eliminando-se o vetor $\Delta y$, obtemos então o sistema de equações diferenciais ordinárias linear

$$
\Delta \dot{x}=A \Delta x,
$$

em que $A=J_{1}-J_{2} J_{4}^{-1} J_{3}$ representa a matriz de estado do sistema, cujos autovalores fornecem informação para análise de estabilidade do sistema. Em geral, a matriz jacobiana é muito grande e esparsa e a matriz de estado, grande e densa. Assim, costuma-se abordar esse problema a partir de sua jacobiana, ou seja, resolver o problema de autovalores generalizados

$$
J z=\lambda B z
$$

em que $J$ é a jacobiana e $B$ é uma matriz diagonal, com 1 nas posições relativas às variáveis de estado e 0 nas relativas às variáveis algébricas.

Vamos fazer agora uma comparação entre o DPSE e dois outros métodos de decomposição parcial do espectro de uma matriz: o método de potências inversas com deslocamento (INVIT) e o método de Arnoldi. Os testes que relataremos a 
Tabela 1: DPSE: sistema sul-sudeste

\begin{tabular}{|r|l|r|}
\hline valor convergido & iter & tempo \\
\hline$-6.3898-7.3828 \mathrm{i}$ & $3(10)$ & 9.67 \\
$-6.8975-6.1249 \mathrm{i}$ & $5(1)$ & 15.43 \\
$-6.8975+6.1249 \mathrm{i}$ & $6(6)$ & 18.03 \\
$-0.0769-6.9928 \mathrm{i}$ & $7(7)$ & 20.30 \\
$-0.2197+6.4944 \mathrm{i}$ & $7(8)$ & 20.30 \\
\hline
\end{tabular}

\begin{tabular}{|r|l|r|}
\hline valor convergido & iter & tempo \\
\hline$-6.3898+7.3828 \mathrm{i}$ & $7(9)$ & 20.31 \\
$-1.0775-0.0000 \mathrm{i}$ & $8(2)$ & 21.59 \\
$-0.0119+3.5121 \mathrm{i}$ & $8(5)$ & 21.60 \\
$-0.1323+5.2125 \mathrm{i}$ & $14(3)$ & 25.54 \\
$-0.0769+6.9928 \mathrm{i}$ & $16(4)$ & 26.22 \\
\hline
\end{tabular}

seguir foram feitos partindo-se de dois modelos: um, com a matriz de estado referente ao sistema sul-sudeste modelado por 50 geradores e 616 barras; outro, com a matriz jacobiana do sistema norte-sul, com 2370 barras, 123 máquinas síncronas e vários outros equipamentos. Ambos os modelos são efetivamente usados na prática e foram gentilmente cedidos pelo CEPEL/Eletrobrás. Para o primeiro modelo, resolvemos o problema de autovalores $A x=\lambda x$, em que $A$ é uma matriz pequena, de ordem 363. Esse modelo reduzido está sendo utilizado aqui para compararmos os resultados obtidos pela nossa implementação do DPSE com os obtidos pelo CEPEL (estes estão reportados em [7]). O segundo modelo dá origem a uma matriz jacobiana $13309 \times 13309$, com 1676 variáveis de estado. Essa matriz tem apenas 48873 elementos não nulos. Nesse caso, fizemos a abordagem do sistema aumentado, isto é, o problema de autovalor generalizado $J z=\lambda B z$.

Os programas para o DPSE e para o INVIT foram desenvolvidos em MATLAB; para o método de Arnoldi, utilizamos a função eigs, que compõe o spar sekit do MATLAB. Para o DPSE, acrescentamos ao critério de convergência do CEPEL $\|(J-\lambda B) z\|<0.00001$, em que $z$ é o resultado (já normalizado, $\|z\|_{2}=1$ ) da resolução do sistema $(J-\lambda B) z=b$, e $\lambda$ o valor calculado por DPSE, a exigência análoga para o autovetor (generalizado) à esquerda.

Para o primeiro modelo, os vetores $b, c$ do DPSE utilizados foram os vetores que definiam uma função de transferência em [7]. Para o segundo modelo, tomamos $b=c=(1 \cdots 1)^{T}$, ou seja, todas as variáveis dinâmicas ligadas. Os valores de saída para os autovalores foram os imaginários puros $i, i * 2, \ldots, i * 10$. Nas tabelas, iter corresponde à iteração em que o valor que está entre parênteses convergiu. Isso nos dá uma idéia do custo computacional do método. Para o primeiro modelo, a convergência para o último autovalor encontrado demandou $3 \times 10+2 \times 9+1 \times 8+$ $1 \times 7+1 \times 4+6 \times 2+2 \times 1=81$ fatorizações LU da matriz $A ; 103$ foi o número de fatorizações no segundo modelo. Isso porque, uma vez que houve convergência para algum autovalor, os vetores $x$ e $y$ (os autovetores à direita e à esquerda, respectivamente) correspondentes ficam fixos para a próxima iteração (lembrando, DPSE é, simplesmente, a iteração sucessiva: espectro de $\left(Y^{T} X\right)^{-1}\left(Y^{T} A X\right)$.

Calculando os resíduos dos autovalores encontrados, em relação à função $F(s)=$ $c^{T}(A-s I)^{-1} b$, conclui-se que os autovalores que correspondem ao maior resíduo são $-6.3898 \pm 7.3828 i$, seguidos de $-0.0769 \pm 6.9928$ e de $-6.8975 \pm 6.1249$ (os resíduos são $c^{T} x y^{T} b$, em que $x$ e $y$ são, respectivamente, os autovetores à direita e à esquerda de $A$ relativos ao autovalor $\lambda, y^{T} x=1$. Ambos são calculados por DPSE). As magnitudes relativas são $1,0.133$ e 0.029 , o que significa que os dois primeiros pares 
Tabela 2: DPSE: sistema norte-sul

\begin{tabular}{|r|l|r|}
\hline valor convergido & iter & tempo \\
\hline$-0.1158+0.2445 \mathrm{i}$ & $7(1)$ & 30.03 \\
$-5.5599+7.7514 \mathrm{i}$ & $7(10)$ & 30.60 \\
$-0.8185+0.5909 \mathrm{i}$ & $8(7)$ & 34.03 \\
$-6.2743+3.6930 \mathrm{i}$ & $8(9)$ & 34.16 \\
$-3.4340+2.2172 \mathrm{i}$ & $10(3)$ & 39.42 \\
\hline
\end{tabular}$\quad$\begin{tabular}{rl|r|r|}
\hline valor convergido & iter & tempo \\
\hline$-1.9511+6.5194 \mathrm{i}$ & $10(5)$ & 39.54 \\
$-0.4546+4.7054 \mathrm{i}$ & $10(6)$ & 39.60 \\
$-5.8150+4.8703 \mathrm{i}$ & $10(8)$ & 39.67 \\
$-1.4778+8.2550 \mathrm{i}$ & $14(2)$ & 44.02 \\
$-0.3720+6.8998 \mathrm{i}$ & $19(4)$ & 47.55 \\
\hline
\end{tabular}

Tabela 3: INVIT: sistema sul-sudeste

\begin{tabular}{|r|l|r|}
\hline valor convergido & iter & tempo \\
\hline$-3.6107+3.0828 \mathrm{i}$ & $6(7)$ & 15.66 \\
$-3.6050+5.5440 \mathrm{i}$ & $8(5)$ & 20.34 \\
$-8.7591-0.9198 \mathrm{i}$ & $8(9)$ & 20.36 \\
$-3.6107+3.0828 \mathrm{i}$ & $8(10)$ & 20.36 \\
$-0.1323+5.2125 \mathrm{i}$ & $11(6)$ & 25.08 \\
\hline
\end{tabular}

\begin{tabular}{|r|l|r|}
\hline valor convergido & iter & tempo \\
\hline$-0.8244+6.9737 \mathrm{i}$ & $11(8)$ & 25.09 \\
$-0.0769+6.9928 \mathrm{i}$ & $13(4)$ & 27.21 \\
$-1000.1-0.0000 \mathrm{i}$ & $15(3)$ & 28.79 \\
$-1000.1+0.0000 \mathrm{i}$ & $16(1)$ & 29.32 \\
$-1000.1+0.0000 \mathrm{i}$ & $24(2)$ & 31.45 \\
\hline
\end{tabular}

de autovalores de maior magnitude podem reproduzir o comportamento dinâmico de $F$, se os autovalores encontrados por DPSE são efetivamente os de maior dominância em relação a $F$. Nesse caso específico, isto é verdadeiro, conforme mostrado em [7]. De qualquer modo, para o problema de estabilidade, vemos que DPSE detectou autovalores quase à direita do eixo imaginário: -0.0769 -6.9928i, - $0.2197+6.4944 \mathrm{i}$, $-0.1323+5.2125 \mathrm{i},-0.0119+3.5121 \mathrm{i}$. O teste realizado com o segundo modelo foi um típico teste com um método para cálculo de autovalores, pois a natureza do modelo que originou a matriz não foi considerada e os vetores iniciais foram o vetor formado por 1's. Observemos que o problema de autovalores generalizados $J z=\lambda B z$ é equivalente ao problema $A x=\lambda x$, para autovalores finitos (ver [2]).

Nas tabelas 3 e 4 listamos os resultados dos testes com INVIT, utilizando-se as mesmas estimativas iniciais de autovalores e de (respectivos) autovetores à direita. Agora, o critério de convergência restringiu-se a $\|(A-\lambda I) v\|<10^{-5}$, com $\|v\|=1$.

Sem algum procedimento de deflação, pode haver repetição de valores convergidos no INVIT, como pode ser visto nos resultados com os dois modelos. O custo

Tabela 4: INVIT: sistema norte-sul

\begin{tabular}{|r|l|r|}
\hline valor convergido & iter & tempo \\
\hline$-0.3181+1.0441 \mathrm{i}$ & $7(1)$ & 49.44 \\
$-2.1087+1.3985 \mathrm{i}$ & $7(10)$ & 49.87 \\
$-3.9967+4.2151 \mathrm{i}$ & $8(2)$ & 55.28 \\
$-3.0522+4.8122 \mathrm{i}$ & $8(4)$ & 55.38 \\
$-3.0522+4.8123 \mathrm{i}$ & $8(5)$ & 55.43 \\
\hline
\end{tabular}

\begin{tabular}{|r|l|r|}
\hline valor convergido & iter & tempo \\
\hline$-1.9511+6.5194 \mathrm{i}$ & $9(6)$ & 59.01 \\
$-0.9113+7.6649 \mathrm{i}$ & $9(8)$ & 59.11 \\
$-1.4778+8.2550 \mathrm{i}$ & $9(9)$ & 59.16 \\
$-1.4778+8.2550 \mathrm{i}$ & $13(7)$ & 64.90 \\
$-1.4778+8.2550 \mathrm{i}$ & $14(3)$ & 65.62 \\
\hline
\end{tabular}


Tabela 5: Arnoldi: sistema sul-sudeste

\begin{tabular}{|r|l|}
\hline valor convergido & iter \\
\hline$-0.3630+0.9869 \mathrm{i}$ & 2 \\
$-0.3630+0.9869 \mathrm{i}$ & 3 \\
$-0.0119+3.5121 \mathrm{i}$ & 1 \\
$-0.0119+3.5121 \mathrm{i}$ & 1 \\
$-0.1323+5.2125 \mathrm{i}$ & 1 \\
\hline \hline & \\
\hline
\end{tabular}

\begin{tabular}{|r|l|}
\hline valor convergido & iter \\
\hline$-0.1482+6.0999 \mathrm{i}$ & 1 \\
$-0.0769+6.9928 \mathrm{i}$ & 1 \\
$-0.5057+7.9522 \mathrm{i}$ & 2 \\
$-0.7073+8.8708 \mathrm{i}$ & 3 \\
$-0.8847+9.8994 \mathrm{i}$ & 4 \\
\hline \hline tempo total & 9.47 \\
\hline
\end{tabular}

computacional, sem deflação, para o método das potências inversas, foi, no primeiro modelo, de $6+8+8+8+11+11+13+15+16+24=120$ fatorizações LU, ou seja, 39 fatorizações LU a mais que DPSE; no segundo modelo, foram feitas 92 fatorizações, 11 a menos que DPSE. Porém, no DPSE, após 90 fatorizações, 8 autovalores já haviam sido calculados. Se relaxarmos o critério de convergência do DPSE, exigindo-se apenas o resíduo do autovetor à direita ser menor que $10^{-5}$, que é como o CEPEL faz, os números devem se aproximar. A deflação no DPSE é intrínseca ao processo: uma vez que o método convergiu para alguns autovetores à direita e à esquerda, eles continuam a exercer pressão sobre os outros vetores, que ainda não convergiram, para que se desviem do espaço invariante gerado por aqueles, no momento da inversão de $Y^{T} X$ Tanto no DPSE e no INVIT, poderíamos ter efetuado mais potências inversas antes de mudarmos os deslocamentos (que são aproximações de autovalores), o que chamamos de pré-multiplicações. Assim, os vetores resultantes teriam se aproximado mais rapidamente de algum subespaço dominante, o que diminuiria o tempo total de execução.

Os testes com o método de Arnoldi foram feitos com a função eigs do MATLAB, que utiliza ARPACK [6]. Essa função computa uma ortonormalização de uma base de Krylov, composta de 20 vetores, o que resulta numa matriz $Q$, com 20 colunas ortonormais, tal que $A Q=Q H+r e_{20}^{T}$, em que $H$ é uma matriz de Hessemberg, $20 \times 20, r$ é o chamado vetor resíduo, e $e_{20}$ é o vetor canônico que tem 1 na última posição (a vigésima). Para cada teste, foram feitas 10 chamadas da forma $\operatorname{eigs}(A, 1, k * i$, opts $)$, para o primeiro modelo, ou da forma eigs $(A, B, 1, k * i$, opts $)$, para o segundo modelo, em que $k$ varia de 1 a 10. A opção opts é para calcular qual a tolerância a ser utilizada para o vetor resíduo. Para realizarmos uma comparação do DPSE e do INVIT com esse método, tivemos que ajustar esse parâmetro de tolerância (que é para controlar a norma do vetor resíduo) para que se adaptasse ao utilizado nos outros dois métodos, e isso foi feito por tentativas, até acharmos a menor potência de 10 tal que $\|(A-\lambda I) x\|<10^{-5}$ ou $\|(A-\lambda B) x\|<10^{-5},\|x\|_{2}=1$. O tempo de execução do método de Arnoldi, aplicado ao primeiro modelo (cuja matriz de estado é pequena), foi bem pequeno e isto deve-se à característica de prémultiplicações do método para obtenção da base de Krylov, além da programação otimizada. Porém, o tempo de execução da função no problema generalizado do segundo modelo foi alto, comparado com o DPSE. 
Tabela 6: Arnoldi: sistema norte-sul

\begin{tabular}{|r|l|}
\hline valor convergido & iter \\
\hline$-0.3179+1.0437 \mathrm{i}$ & 1 \\
$-0.3905+1.6871 \mathrm{i}$ & 1 \\
$-0.5199+2.8814 \mathrm{i}$ & 1 \\
$-0.5551+4.1191 \mathrm{i}$ & 1 \\
$-0.4546+4.7054 \mathrm{i}$ & 1 \\
\hline \hline & \\
\hline
\end{tabular}

\begin{tabular}{|r|l|}
\hline valor convergido & iter \\
\hline$-0.5775+6.2566 \mathrm{i}$ & 2 \\
$-0.3720+6.8998 \mathrm{i}$ & 1 \\
$-0.6274+7.7113 \mathrm{i}$ & 3 \\
$-0.6589+9.1398 \mathrm{i}$ & 2 \\
$-0.7901+10.0372 \mathrm{i}$ & 2 \\
\hline \hline tempo total & 57.03 \\
\hline
\end{tabular}

\title{
5. Conclusão
}

Atualmente, descobrem-se cada vez mais conexões de métodos de resolução de espectro com métodos tipo Newton. O método de Jacobi-Davidson é um outro exemplo recente [11], [12]. Resultados obtidos com processamento paralelo do DPSE e comparações do DPSE com outros métodos (além dos já citados, uma versão de um método desenvolvido em [5]) também podem ser vistos em [3].

\begin{abstract}
In this paper a numerical analysis of some partial eigensolution methods is developed by showing an actual relationship of these methods with the calculus of zeros of functions by Newton's method. One of these methodos is DPSE (Dominant Pole Spectrum Eigensolver), arisen in Control. DPSE, which is a Newton-type method, has local quadratic convergence and this fact is proved here. Also, interesting results obtained from DPSE in small-signal stability snalysis of the Brazilian power system are shown and compared with the results obtained by standard methods utilized for this problem.
\end{abstract}

\section{Referências}

[1] R. Bellman, "Introduction to Matrix Analysis", 2nd ed., SIAM, Philadelphia, 1997.

[2] L.H. Bezerra e C. Tomei, Spectral Transformation Algorithms for Computing Unstable Modes, Comp. Appl. Math., 18 (1999), 1-14.

[3] L.H. Bezerra, C. Tomei e R.A. McCoy, Möbius Transforms and Solvers for Large Sparse Generalized Nonsymmetric Eigenvalue Problems, Tech. Rep. TR/PA/98/03, CERFACS, Toulouse, 1998.

[4] S. Gomes Jr., N. Martins e C. Portela, Computing Small-Signal Stability Boundaries for Large-Scale Power Systems, IEEE Trans. on Power Systems, 18, No. 2 (2003), 747-752.

[5] A. Jennings e W.J. Stewart, Simultaneous Iteration for Partial Eigensolution of Real Matrices, J. Inst. Math. Appl., 15 (1975), 351-361. 
[6] R.B. Lehoucq, D.C. Sorensen e C. Yang, "ARPACK Users' Guide: Solution of Large Scale Eigenvalue Problems with Implicitly Restarted Arnoldi Methods", SIAM, Philadelphia, 1998.

[7] N. Martins, The Dominant Pole Spectrum Eigensolver, IEEE Trans. on Power Systems, 12, No. 1 (1997), 245-254.

[8] N. Martins, L.T.G. Lima e H.J.C.P. Pinto, Computing Dominant Poles of Very High Order Transfer Functions, IEEE Trans. on Power Systems, 11, No. 1 (1996), 162-170.

[9] N. Martins e P.E.M. Quintão, Computing dominant poles of power system multivariable transfer functions, IEEE Trans. on Power Systems, 18, No. 1 (2003), 152-159.

[10] G. Peters e J.H. Wilkinson, Inverse Iteration, Ill-Conditioned Equations and Newton's Method, SIAM Rev., 21 (1979), 339-360.

[11] G.L.G. Sleijpen e H.A. Van der Vorst, A Jacobi-Davidson iteration method for linear eigenvalue problems, SIAM Rev., 42 (2000), 267-293.

[12] G.L.G. Sleijpen e H.A. Van der Vorst, The Jacobi-Davidson method for eigenvalue problems and its relation with accelerated inexact Newton schemes, em "Iterative Methods in Linear Algebra, II" (S.D. Margenov e P.S. Vassilevski, eds.), IMACS Ser. Comput. Appl. Math., 3, New Brunswick, NJ, (1996) 377389. 
